\title{
PHYSICAL MODELING OF FLOW IN A TUNDISH FOR CONTINUOUS CASTING MACHINE
}

The paper describes a procedure for obtaining similarity criteria for a real object - a tundish for continuous casting machine (CCM) and its reduced physical model. The procedure is demonstrated on the basis of Navier-Stockes differential equations and with the help of dimensional analysis. To obtain similarity criteria dimensional analysis makes use of dimensions of those physical quantities which significantly influence the mentioned phenomenon. From the above it results that when modeling a concrete phenomenon with the help of this method it is inevitable to thoroughly know the physical substance of the phenomenon to expressly ensure the choice of all relevant quantities. On the basis of elaborated model laws the basic characteristics of flow (rate, flow volume, time intervals) were designed for the reduced model of the real CCM. The obtained dependences of characteristics feature the universal validity for all the models of tundishes.

Keywords: liquid iron, flow, modeling, similarity criteria.

\section{Introduction}

At continuous casting of steel the full ladle is put on a rotating turret of the casting machine. The ladle shroud having been set and ladle slide gate having been opened, the tundish starts being filled until the required surface height is achieved [1]. Discharge from the tundish to the mould is in the case of submerged casting usually stopper controlled; in the case of open stream casting it is given by the diameter of the nozzle and casting speed. The tundish is one of the basic parts of the casting machine as it fulfils various important functions. Its size is usually $8-10 \%$ of the ladle. In the case of CCM for billets steel from one tundish is distributed into as many as six molds. When there are more strands, the casting machine makes use of two tundishes [2, 3 and 4]. The requirements put on the tundish result into different demands put on its shape, arrangement as well as size due to the fact that some of the required functions oppose each other. For example, more perfect homogenization of chemical composition and temperature of steel are achieved at intensive mixing of the molten metal; on the other hand, possibilities of inclusions washing-out and their subsequent trapping in cover slag are getting worse. Although a larger volume of the tundish contributes to a better separation of non-metallic inclusions, it also prolongs non-stationary states at exchanges of a ladle [5, 6, 7 and 8].

The authors are not aware of the fact that the complicated phenomenon of steel flow modeling in the tundish in reduced physical models has been described on the basis of criterion equations in such a way as it is presented in this paper. General information on modeling can be found in some publications [9, 10 and 11]. This article presents the flow process in a tundish analyzed by means of similarity criteria characterizing the most general fluid motion. Consequently, the criteria that are irrelevant for steel flow in the tundish were omitted [12]. All similarity criteria will be derived by means of two methods - from differential equations and on the basis of dimensional analysis [13,14, 15 and 16].

\section{Analytical method of determining similarity criteria from Navier-Stokes equations}

Navier-Stokes differential equations are the most general equations of unsteady spatial isothermal motion of real, i. e., viscous fluid. They express the equilibrium of unit forces of mass $(a)$, pressure $(\partial p /(\rho \cdot \partial y))$, friction $\left(v \cdot \nabla^{2} v\right)$ and inertia $\left(\mathrm{D} v_{y} / \mathrm{d} \tau\right)$. Making use of so called complete (total, substitution) derivation of velocity components and referring forces to the unit of mass, we get the below relation for the axis $x$

$$
a_{x}-\frac{1}{\rho} \cdot \frac{\partial p}{\partial x}+\nu \cdot \nabla^{2} v_{x}=\frac{\mathrm{D} v_{x}}{\mathrm{~d} \tau}\left(\mathrm{m} \cdot \mathrm{s}^{-2}\right)
$$

Similar relations hold for two other coordinate axes. In the vector record it is the relation in the form

$$
\boldsymbol{a}-\frac{1}{\rho} \cdot \boldsymbol{g r a d} p+\nu \cdot \nabla^{2} \boldsymbol{v}_{x}=\frac{\mathrm{D} v}{\mathrm{~d} \tau}\left(\mathrm{m} \cdot \mathrm{s}^{-2}\right)
$$

Considering the most common case of force field of the Earth's gravity, then acceleration $a$ in equation (2) is replaced by gravitational acceleration $g$, or by its components. Equation (2) has to hold both for the model and for the real object. When equation (1) is rewritten with appropriate similarity constants of quantities " $c$ " (quantity in the real object D to the magnitude in the model $\mathrm{M}$ ) we get the below relation for the axis $x$

\footnotetext{
* Maria Carnogurska ${ }^{1}$, Miroslav Prihoda ${ }^{2}$

${ }^{1}$ Department of Power Engineering, Faculty of Mechanical Engineering, Technical University of Kosice, Slovakia, E-mail: maria.carnogurska@tuke.sk

${ }^{2}$ Faculty of Metallurgy and Materials Engineering, VSB - Technical University of Ostrava, Ostrava-Poruba, Czech Republic
} 


$$
c_{g} \cdot a_{y}-\frac{c_{p}}{c_{\rho} \cdot c_{l}} \cdot \frac{1}{\rho} \cdot \frac{\partial p}{\partial y}+\frac{c_{\nu} \cdot c_{v}}{c_{l}^{2}} \cdot \nu\left(\frac{\partial^{2} v_{y}}{\partial x^{2}}+\frac{\partial^{2} v_{y}}{\partial y^{2}}+\frac{\partial^{2} v_{y}}{\partial z^{2}}\right)=\frac{c_{v}}{c_{\tau}} \cdot \frac{\partial v_{y}}{\partial \tau}+\frac{c_{v}^{2}}{c_{l}}\left(v_{x} \frac{\partial v_{y}}{\partial x}+v_{y} \frac{\partial v_{y}}{\partial y}+v_{z} \frac{\partial v_{y}}{\partial z}\right)
$$

where $c_{g}$ - scale of change in gravitational acceleration, $c_{p}$ - scale of change in pressure, $c_{\rho}$ - scale of change in density, $c_{l}$ - scale of change in length, $c_{v}$ - scale of change in kinematic viscosity, $c_{v}$ scale of change in velocity.

Similar equation can be written for two other axes. When equation (3) is divided by member $c_{v}^{2} / c_{l}$, we get the relation and similarly also for the axes $x$ and $z$. ilarity of the real object to its reduced model only when starting equations will have an unambiguous solution. It can be achieved only when we add to the system of differential equations such conditions which will lead to an only solution expressly characterizing the observed phenomenon, i. e. conditions of one single meaning. The conditions have to contain geometry of space, physical properties of the ambient in which the observed phenomenon develops as well as values of variable quantities on the boundary of the

$$
\frac{c_{g} \cdot c_{l}}{c_{v}^{2}} \cdot a_{y}=\frac{c_{p}}{c_{\rho} \cdot c_{v}^{2}} \cdot \frac{1}{\rho} \cdot \frac{\partial p}{\partial y}+\frac{c_{\nu}}{c_{\nu} \cdot c_{l}} \cdot \nu\left(\frac{\partial^{2} v_{y}}{\partial x^{2}}+\frac{\partial^{2} v_{y}}{\partial y^{2}}+\frac{\partial^{2} v_{y}}{\partial z^{2}}\right)=\frac{c_{l}}{c_{v} \cdot c_{\tau}} \cdot \frac{\partial v_{y}}{\partial \tau}+\left(v_{x} \frac{\partial v_{y}}{\partial x}+v_{y} \frac{\partial v_{y}}{\partial y}+v_{z} \frac{\partial v_{y}}{\partial z}\right)
$$

Equation (4) holds for all groups of mutually similar hydrodynamic phenomena. As long as equations (1) and (4) are to represent both the model and reality, they have to be identical [10]. For indicators of model and real object similarities the following equation can be written

$$
\frac{c_{l}}{c_{\tau} \cdot c_{\nu}}=\frac{c_{g} \cdot c_{l}}{c_{v}^{2}}=\frac{c_{p}}{c_{\rho} \cdot c_{\nu}^{2}}=\frac{c_{\nu}}{c_{\nu} \cdot c_{l}}=1
$$

From the indicators four similarity criteria can be derived in the form

$$
\pi_{1}=\frac{l}{\tau \cdot v}, \pi_{2}=\frac{g \cdot l}{v^{2}}, \pi_{3}=\frac{p}{\rho \cdot v^{2}}, \pi_{4}=\frac{\nu}{v \cdot l}
$$

Individual criteria have the following meaning

$$
\pi_{1}=\frac{l}{\tau \cdot v}=H o^{-1}
$$

reciprocal of homochronism criterion (Strouhal's number) (7)

$$
\pi_{2}=\frac{g \cdot l}{v^{2}}=F r^{-1}
$$

reciprocal of the Froude criterion

$$
\pi_{3}=\frac{p}{\rho \cdot v^{2}}=E u
$$

Euler's criterion

$$
\pi_{4}=\frac{\nu}{v \cdot l}=R e^{-1}
$$

reciprocal of the Reynolds criterion

The general criterion equation of spatial isothermal motion of incompressible viscous fluid derived from Navier-Stokes differential equations has then the following form

$$
f(H o, F r, E u, R e)=0
$$

The system of differential Navier-Stokes equations expresses a whole group of phenomena and that is why it has an indefinite number of solutions. Equation (11) can express a mechanical sim- system and in the initial moment of the observed phenomenon. Boundary conditions have to guarantee constant values of similarity constants for each of variable quantities occurring on the boundary of the process. As for the initial conditions it is necessary to keep constant values of constants of proportion in all relevant quantities of the phenomenon. When comparing two phenomena we find out that similarity indicators formed from similarity constants are equal to one, such phenomena can be referred to as similar.

\section{Analytical method of deriving similarity criteria with the help of dimension analysis}

Physical quantities that influence isothermal motion of fluid are:

- kinematic viscosity $v\left(\mathrm{~m}^{2} \cdot \mathrm{s}^{-1}\right)$,

- density $\rho\left(\mathrm{kg} \cdot \mathrm{m}^{-3}\right)$,

- rate of flow $v\left(\mathrm{~m} \cdot \mathrm{s}^{-1}\right)$,

- gravitational acceleration $g\left(\mathrm{~m} \cdot \mathrm{s}^{-2}\right)$,

- length $l(\mathrm{~m})$,

- pressure $p\left(\mathrm{~kg} \cdot \mathrm{m}^{-1} \cdot \mathrm{s}^{-2}\right)$,

- time $\tau$ (s).

The complete physical equation characterizing dependence of mentioned physical quantities has the following form

$$
\varphi(v, \rho, v, g, l, p, \tau)=0
$$

Equation (12) is dimensionally homogeneous; therefore, individual physical quantities cannot occur in it independently, only in the form of products $[15,16]$

$$
\pi_{i}=v^{x_{1}} \cdot \rho^{x_{2}} \cdot v^{x_{3}} \cdot g^{x_{4}} \cdot l^{x_{5}} \cdot p^{x_{6}} \cdot \tau^{x_{7}}
$$

where exponents $x_{1}, x_{2}, \ldots, x_{7}$ are unknown and will have to be determined later.

Dimensional matrix for the basic unit will consist of $n=7$ columns and a total of $m=3$ lines and has the form 


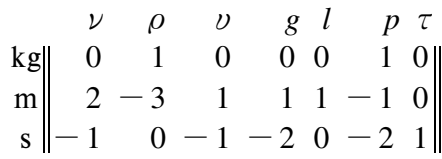

When the matrix rank $h=3$ and number of relevant quantities $n=7$ it is possible to create the total of $l=n-h$, i. e. 4 dimensionless arguments $\pi$. For calculation of unknown $\left(x_{i}>m\right)$ the rectangular dimensional matrix $\mathrm{A}$ is divided into two parts; similarly we proceed when dividing the vector of unknown quantities $x_{i}$. The first part of the matrix is square with a number of $h$ columns and $h$ lines, while the matrix columns have to be chosen so that its determinant will be nonzero $(\Delta \neq 0)$. This also complies with division of vectors $x_{i}$.

The shape of square matrix A and vector of unknown quantities $x_{i}$ from equation (14) will be arranged and written in a simplified form

$$
\mathbf{A} \cdot \mathbf{B}=(-1) \cdot \mathbf{D} \cdot \mathbf{E}
$$

Matrix (15) in an itemized form for individual dimensions is as follows

$$
\left\|\begin{array}{rrr}
1 & 0 & 0 \\
-3 & 1 & 1 \\
0 & -1 & 0
\end{array}\right\| \cdot\left\|\begin{array}{l}
x_{2} \\
x_{3} \\
x_{5}
\end{array}\right\|=(-1)\left\|\begin{array}{rrrr}
0 & 0 & 1 & 0 \\
2 & 1 & -1 & 0 \\
-1 & -2 & -2 & 1
\end{array}\right\| \cdot\left\|\begin{array}{l}
x_{1} \\
x_{4} \\
x_{6} \\
x_{7}
\end{array}\right\|
$$

Determinant of matrix A is determined, for example, on the basis of Laplace development and has to $b e \neq 0$.

$$
\Delta_{\mathrm{A}}=\Sigma(-1)^{i+j}=a_{i j} \cdot M_{i j}
$$

where

$a_{i j}$ is the element in the ith line and jth column,

$M_{i j}$ - subdeterminant of the matrix in which the $i$ th line and $j$ th column are omitted. The value of matrix determinant is $\Delta_{\mathrm{A}}=1$, by which the solvability condition has been fulfilled.

Choice of redundant unknowns $x_{1}, x_{4}, x_{6}, x_{7}$ is repeated four times paying attention to the fact that the choices have to be linearly independent. The selection matrix has the following form

\begin{tabular}{lllll} 
& $\mathrm{x}_{1}$ & $\mathrm{x}_{4}$ & $\mathrm{X}_{6}$ & $\mathrm{x}_{7}$ \\
\hline 1st choice & 1 & 0 & 0 & 0 \\
2nd choice & 0 & 1 & 0 & 0 \\
3rd choice & 0 & 0 & 1 & 0 \\
4th choice & 0 & 0 & 0 & 1
\end{tabular}

and its determinant has the value $\Delta=1$, by which the solvability condition has been fulfilled.

Transformation of equation (16) to the system of linear equation is in the form

$$
\begin{aligned}
x^{2} & =-x_{6} \\
-3 \cdot x_{2}+x_{3}+x_{5} & =-2 \cdot x_{1}-x_{4}+x_{6} \\
-x_{3} & =x_{1}+2 \cdot x_{4}+2 \cdot x_{6}-x_{7}
\end{aligned}
$$

Solving the system of linear equations (19) we get the following similarity criteria

\begin{tabular}{l|rrrrrrr} 
& $x_{1}$ & $x_{2}$ & $x_{3}$ & $x_{4}$ & $x_{5}$ & $x_{6}$ & $x_{7}$ \\
\hline$\pi_{1}$ & 1 & 0 & -1 & 0 & -1 & 0 & 0 \\
$\pi_{2}$ & 0 & 0 & -2 & 1 & 1 & 0 & 0 \\
$\pi_{3}$ & 0 & -1 & -2 & 0 & 0 & 1 & 0 \\
$\pi_{4}$ & 0 & 0 & 1 & 0 & -1 & 0 & 1
\end{tabular}

Dimensionless arguments themselves have the form

$$
\pi_{1}=\frac{\nu}{v \cdot l}, \pi_{2}=\frac{g \cdot l}{v^{2}}, \pi_{3}=\frac{p}{\rho \cdot v^{2}}, \pi_{4}=\frac{\tau \cdot v}{l} .
$$

From the way in which the criteria are derived results that all the expressions in (21) are dimensionless numbers, which can be easily verified by substituting dimension of individual quantities. Dimensionless form of the function describing steel flow can be written in the form

$$
\varphi\left(\pi_{1}, \pi_{2}, \pi_{3}, \pi_{4}\right)=0
$$

The acquired criteria in equation (22) one after the other are: reciprocal of the Reynolds criterion $R e^{-1}$, reciprocal of the Froude criterion $\mathrm{Fr}^{-1}$, Euler's criterion $\mathrm{Eu}$ and homochronism criterion $H o$, and expression (22) can also be written as

$$
\varphi(R e, F r, E u, H o)=0
$$

As can be seen from comparison of relations (23) a (11) when looking for conditions of mechanical similarity of flow with the help of dimension analysis, two identical similarity numbers were achieved equally as when using the analysis method of NavierStokes differential equations.

\section{Similarity between the model and real object}

Physical similarity between the model and real object should be guaranteed by equal similarity criteria both in the real object and model. In the case of a steel flow simulation in the tundish four similarity criteria were derived - equations (21) or (23).

\section{Homochronism criterion}

also known as Strouhal's criterion $(S h)$, which apart from inertia force contains also impulse force, i. e. force related to the change of motion, is used for expression of dimensionless time. It can also be understood in such a way that the relative unit of measurement is time which fluid particles moving at the speed $v$ need to cover the path $l$. If it refers to the process which is stationary, i. e. the 
process at the constant speed, the criterion Ho becomes irrelevant and equation (23) will be simplified and will have the form

$$
\varphi(\operatorname{Re}, F r, E u)=0
$$

\section{Euler's criterion}

that includes forces of pressure and inertia serves for determination of pressure losses in the forced flow. This similarity number is significant especially when solving flow in pipes. For the flow in open channels, which is the case of motion of molten steel in the tundish, pressure losses are negligible. The Euler's number can therefore be omitted from relation (23) and the criterion equation gets the form

$$
\varphi(R e, F r)=0
$$

\section{Reynolds criterion}

expressing the ratio of inertia to friction forces characterizes the flow of real liquid which is either laminar or turbulent. Transition from laminar to turbulent flow occurs at the critical value of the Reynolds criterion denoted $R e_{\mathrm{kr}}$. If for a particular case $R e>R e_{\mathrm{kr}}$, the flow is turbulent; if $R e<R e_{\mathrm{kr}}$ the flow is laminar.

The similarity condition between the model (M) and real object (D) is by means of this criterion expressed as follows

$$
R e_{M}=R e_{D}, \text { or } R e=R e^{\prime}
$$

The similarity condition for the real object (tundish) and model (reduced tundish) - relation (26) - can be written as follows

$$
\frac{v \cdot l}{\nu}=\frac{v^{\prime} \cdot l^{\prime}}{\nu^{\prime}}
$$

The below mentioned expression must hold for the similarity constants

$$
\frac{c_{v}}{c_{\nu}}=c_{l}^{-1}
$$

If the kinematical viscosity of liquids both in the real object and in the model is identical, i. e., if $c_{v}=1$, then equation (27) is simplified to the form

$$
c_{v}=c_{l}^{-1}
$$

For the model of the tundish ten times smaller than the real object holds that the scale of change in length is $c_{l}=10$. To satisfy equation (28) it has to be $c_{v}=0.1$, which means that velocity in the model diminished in this way would be ten times greater than the one in the real object. If the model fluid had ten times smaller kinematical viscosity than the real object liquid, the following would hold $c_{v}=1$, i. e., velocity in the model would be equal to the real object velocity.

\section{Froude criterion}

represents the ratio of inertia to mass forces. If the equality of number $F r$ is kept at modeling, then the following holds
$F r_{\mathrm{D}}=F r_{\mathrm{M}}$ resp. $\frac{v^{\prime 2}}{g^{\prime} \cdot l^{\prime}}=\frac{v^{2}}{g \cdot l}$

For particular similarity constants we then get

$$
c_{v}^{2}=c_{g} \cdot c_{l}
$$

The processes in the model as well as those in the real object will take place under earth gravitation, i. e.

$$
g=g \Rightarrow c_{g}=1
$$

so equation (30) then has, at the similarity constant of gravitation acceleration $c_{g}=1$, the form

$$
c_{v}^{2}=c_{l} \text { resp. } \quad c_{v}=\sqrt{c_{l}}=c_{l}^{1 / 2}
$$

If the Froude criterion is applied to the model in the same diminishment as the Reynolds criterion, i. e. in the scale 10:1, the rates in the model are 3.16 times smaller than those in the real object. Comparing the conditions of Froude and Reynolds similarities we can see that the modeling of motion phenomena at keeping number $\mathrm{Fr}$ is substantially simpler because the required rates of flow in the model are lower than those in the real object. When modeling according to $R e$ the situation is opposite. Similar unfavorable ratio is also in volume flows, which often leads to serious technical problems. Comparing relations (28) and (31) we can see that both of them can simultaneously hold only at the length scale $c_{l}=1$, i. e. in the case when both the model and real object have the same dimensions.

When using in the reduced model of the tundish the fluid with the same kinematical viscosity as the one used in the real object, the below condition cannot be satisfied

$$
R e^{\prime}=\operatorname{Re} \wedge F r^{\prime}=F r
$$

Theoretically, the current equality of both criteria in the model and in real object can be ensured with the choice of such model liquid for which equation (33) will hold for kinematical viscosity

$$
c_{v}=c_{l} \cdot \sqrt{c_{l}}=c_{l}^{3 / 2}
$$

To achieve the total mechanical similarity between two hydrodynamic phenomena in the modeling research requires the use of such liquid which will guarantee simultaneous satisfaction of equality of all necessary similarity criteria both in the model and in the real object [17]. However, it is impossible to find two liquids that feature such physical characteristics to satisfy the mentioned conditions. That is why the approximate mechanical similarity has to be used when some criteria are considered irrelevant.

As we have already mentioned, when modeling the flow of liquid steel in the tundish it is possible to omit the criteria $\mathrm{Ho}$ and $E u$. It has also been mentioned that in practice the condition (32), which is simultaneous equality of numbers $R e$ and $F r$, can hardly be satisfied. Concretely, in a ten times smaller model it would be 
necessary to work with modeling fluid which would, in comparison with fluid in the real object, feature 31.6 times smaller kinematical viscosity. For modeling the fluid steel flow it would be required to use fluid featuring kinematical viscosity of order $10^{-8} \mathrm{~m}^{2} \cdot \mathrm{s}^{-1}$. At the same time it has to be said that fluid featuring such kinematical viscosity at usual ambient temperatures practically does not exist

If considering realistic possibilities of choice of model liquid which would, apart from certain physical characteristics, feature an easily accessible price, satisfy hygienic standards, and be easily stored then, it is water that can be used for flow modeling. Another advantage of water also is that its kinematical viscosity at the usual ambient temperature does not differ from kinematical viscosity of molten steel; they both are about the value of $1 \cdot 10^{-6} \mathrm{~m}^{2} \cdot \mathrm{s}^{-1}$.

Referring to the above mentioned facts it is obvious that at modeling the molten steel flow in the tundish for continuous steel pouring machine it is desired to decide which criterion will be chosen as determining - the Froude or Reynolds criterion.

When comparing both similarity criteria we can see that for the physical model of steel flow in tundish for continuous steel pouring machine it is more convenient to prioritize the Froude criterion. Kinematically similar motion phenomena which are exclusively or predominantly influenced by the gravitation force are also dynamically similar when they have the same values $F r$ in mutually corresponding cross sections of both real object and model.

\section{Results and discussion}

Research into flow similarities in a diminished model of the tundish comes out from geometrical similarity as the basis for mechanical similarity. Apart from the similarity constant of velocity expressed by relation (31), which is written in the following way

$$
c_{v}=\frac{v^{\prime}}{v}=c_{l}^{1 / 2}
$$

it is possible to define other similarity constants for the model and the real object, which is the similarity constant of volume flow

$$
c_{Q_{v}}=\frac{Q_{V}^{\prime}}{Q_{V}}=\frac{S^{\prime} \cdot v^{\prime}}{S \cdot v}=c_{l}^{2} \sqrt{c_{l}}=c_{l}^{5 / 2}
$$

and similarity constant of time intervals

$$
c_{\tau}=\frac{c_{l}}{c_{\nu}}=\frac{c_{l}}{\sqrt{c_{l}}}=c_{l}^{1 / 2}
$$

Applying the derived similarity constants of individual quantities to the tundish for a six-strand continuous casting machine (Fig. 1) casting billets having a cross section of $180 \times 180 \mathrm{~mm}$ at the rate of $2.1 \mathrm{~m} \cdot \mathrm{min}^{-1}$ we can obtain the following information.

Mass flow of steel (oc) from the ladle to the tundish is

$$
\begin{aligned}
& Q_{m, \mathrm{oc}}=6 \cdot S \cdot v \cdot \rho=6 \cdot 0.18 \cdot 0.18 \cdot 2.1 \cdot 7800= \\
& =3184 \mathrm{~kg} \cdot \mathrm{min}^{-1}
\end{aligned}
$$

where

$v$ - pouring rate $\left(\mathrm{m} \cdot \mathrm{min}^{-1}\right)$,

$\rho$ - density of steel at normal temperature $\left(\mathrm{kg} \cdot \mathrm{m}^{-3}\right)$,

$S$ - cross section of raw casting (m).

Volume flow of steel from the ladle to the tundish

$$
\begin{aligned}
& Q_{V, \mathrm{oc}}=\frac{Q_{m, \mathrm{oc}}}{\rho_{l}}=\frac{3184}{6900}=0.4615 \mathrm{~m}^{3} \cdot \mathrm{min}^{-1}= \\
& =461.5 \mathrm{l} \cdot \mathrm{min}^{-1}
\end{aligned}
$$

where

$\rho_{t}$ - density of molten steel $\left(\mathrm{kg} \cdot \mathrm{m}^{-3}\right)$.

When a reduced model of a tundish is in the scale 10:1, it will hold that $c_{l}=10$; then, in compliance with equation (35) the volume flow of water (in) into the model is equal to

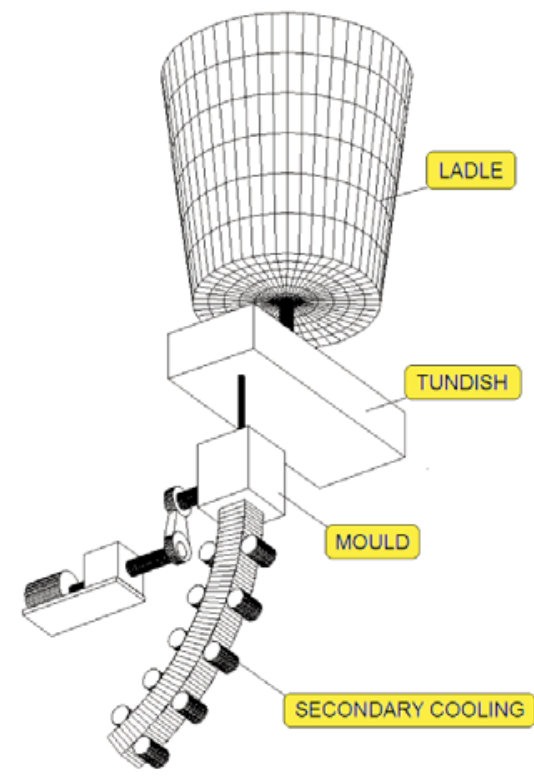

Fig. 1 Schematic diagram of a CCM [18]

$$
Q_{V, \mathrm{vo}}=\frac{Q_{V, \mathrm{oc}}}{c_{l}^{5 / 2}}=\frac{461.5}{10^{5 / 2}}=1.461 \cdot \mathrm{min}^{-1}
$$

and, therefore, it will be 316-times smaller than the volume flow of molten steel in the real tundish (Fig. 2). The place in the figure is marked with a circle on the axis $y$. The figure presents the dependence of the ratios of volume flows on the reduction scale within the scales 10:1 to $1: 1$, which represents the congruence of the model and real object. Water flow rate in the ten times smaller model will be 3.16 times smaller than steel flow rate in the tundish, i. e. its value is $0.66 \mathrm{~m} \cdot \mathrm{s}^{-1}$ and time intervals in the model will be 3.16-times smaller than those in the real tundish. At the same time the figure presents the dependence of the ratios of flow rate and time intervals to the scale of the model reduction within the same boundaries as in volume flows. 


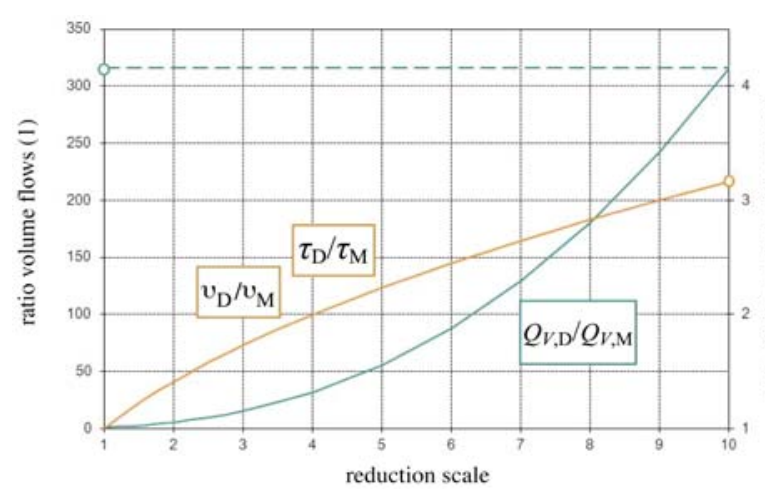

Fig. 2 Dependence of ratio of volume flows, rate and time intervals on the scale of reduction

If the model of the tundish is in the scale, e. g. 8:1, the volume water flow will be $2.551 \cdot \mathrm{min}^{-1}$ (181-times smaller than molten steel flow in the tundish), flow rates will be 2.83-times smaller than the rates of molten steel in the real object and time intervals in the model will be 2.83-times shorter. For the model of the tundish in the scale 5:1 similar values for volume flow will be equal to $8.261 \cdot \mathrm{min}^{-1}$ (55.9-times shorter than the volume flow of molten steel in the tundish), flow rates will be 2.24-times smaller and times will be 2.24-times shorter. If the model is designed in other scale of lengths, the scale for other quantities will be determined from Fig. 2.

\section{Conclusions}

There is no doubt about advantages of modeling of continuous steel casting in laboratory conditions. Approximate mechanical similarity is applied in the presented procedure of isothermal modeling of molten steel flow in the tundish. Water was chosen as the model fluid due to its physical characteristics.

In the paper we present the process of determining volume flow of water in the reduced laboratory model of the tundish for a six-strand continuous machine whose geometrical similarity to the real object was given in the scales from 10:1 to 1:1. Respecting derived similarity constants of individual physical quantities, results of this analysis can be applied also for an arbitrary number of strands in a pouring machine or for two tundishes.

\section{Acknowledgements}

The publication is based on the solution of the partial problem of grant project VEGA No. 1/0006/11, KEGA 045-015TUKE4/2010 and SP2011/29-FMMI VŠB TUO.

\section{References}

[1] STRANSKY, K., KAVICKA, F., SEKANINA, B., STETINA, J., MAUDER, T., STRANSKY, L.: Acta Metallurgica Slovaca, vol. 16, No. 4, 2010, pp. 216-222.

[2] DOLEJSI, Z., REDR, M., PRIHODA, M., MOLINEK, J., JURECKA, P.: Continuous Steel Casting. Prague, TEVUH, 1987, 383 p. (in Czech).

[3] DOLEJSI, Z., PRIHODA, M., MOLINEK, J., JURECKA, P., VESELY, K.: Continuous Steel Casting. $2^{\text {nd }}$, vol. Prague, TEVUH, 1988, 191 p. (in Czech).

[4] PRIHODA, M., BAZAN, J., DOBROVSKA, J., JELINEK, P., JONSTA, Z., VROZINA, M.: New Knowledge from Research into Continuous Steel Casting. Ostrava : VSB-TU Ostrava, 2001, 177 p. (in Czech).

[5] MOLINEK, J., VACLAVIK, L., KLIMSZOVA, A., PRIHODA, M.: Acta Metallurgica Slovaca, vol. 13, No. 5, 2007, pp. 355-360 p. (in Czech).

[6] PRIHODA, M., MOLINEK, J., PYSZKO, R., FOJTIK, P.: Acta Metallurgica Slovaca, vol. 10, No. 3 (special issue), pp. 434-438 (in Czech).

[7] ABOUTALEBI REZA, M., HASAN, M., GUTHRIE, L. I. R.: Metallurgical and Materials Transactions B, 1995, No. 26B, pp. $731-744$.

[8] HUESPE, E. A., CARDONA, A., FACHINOTTI, V.: Computer Methods in Applied Mechanics and Engineering, 2000, No. 182, pp. 439-455.

[8] PASCON, F., HABRAKEN, A., BLES, G.: J. de Physique IV, 2004, vol. 120, pp. 447-455.

[9] PRIHODA, M., MOLINEK, J., DITTEL, D., VACUlIK, M., FOJTIK, P.: Acta Metallurgica Slovaca, vol. 13, 2007, No. 3, pp. 311-316 (in Czech).

[10] PRIHODA, M., JURECKA, P., MOLINEK, J., REDR, M., TLUSTY, R.: Hutnicke listy XXXX, 1985, No. 7, pp. 491-497, (in Czech).

[11] SANTOS, A. C. , SPIM, A. J., GARCIA, A.: Engineering Applications of Artificial Intelligence, vol. 16, No. 16, 2003, pp. 511-527.

[12] CARNOGURSKA, M., PRIHODA, M.: Application of Dimensional Analysis in Energetic. Vienala, Kosice, 2011, 214 p. (in Slovak).

[13] CARNOGURSKA, M., PRIHODA, M., BRESTOVIC, T.: Chemical and Process Engin., vol. 32, No. 3, 2011, pp. 175-184, DOI: 10.2478/v10176-011-0013-7.

[14] CARNOGURSKA, M., PRIHODA, M., POPCAKOVA, D.: J. of Mechanical Science and Technology, vol. 24, 2010, No. 12, pp. 2479-2486.

[15] LANGHAR, H. L.: Dimensional Analysis and Theory of Models. Malabar : Robert E. Kreiger Publishing Company, 1987 (in Czech). 
[16] BARENBLATT, G. I.: Dimensional Analysis. New York : London : Paris : Montreaux : Tokyo: Gordon and Breach Science Publishers, 1987.

[17] VOJSOVICOVA, M., LIPTAKOVA, T., ZATKALIKOVA, V.: Rheological Characteristics of Thermoplastic Polymers after Degradation, Communications: Scientific Letters of the University of Zilina. - ISSN 1335-4205. - vol. 13, No. 4 (2011), pp. 32-35.

[18] PYSZKO, R.: Research into Working Conditions in Crystallizer at Continuous Steel Casting. Doctoral dissertation. VSB-TU Ostrava, 1993, 133 p., (in Czech). 